\title{
Esiti materno-fetali e glicemia a digiuno vs una o due ore dopo il carico orale di glucosio nelle donne con diabete gestazionale
}

\author{
Mariangela Caporusso $^{1}$ - Luigi Laviola ${ }^{1}$
}

Accettato: 1 dicembre 2020 / Pubblicato online: 27 maggio 2021

(c) The Author(s) 2021

Commento a:

Elevated fasting vs post-load glucose levels and pregnancy outcomes in gestational diabetes: a population-based study.

E.A Ryan, A. Savu, R.O. Yeung, L.E. Moore, S.L. Bowker, Kaul P.

Diabetic Med (2020) 37(1):114-122

Il mancato riconoscimento del diabete mellito gestazionale (GDM) e la persistenza di elevati valori glicemici nel corso della gravidanza è associata a esiti materni e fetali negativi [1]. Nello studio Hyperglycemia and Adverse Pregnancy Outcomes (HAPO) è stata individuata un'associazione statisticamente significativa tra gli elevati valori di glicemia e alcuni esiti materno-fetali negativi, come la macrosomia e la preeclampsia, senza tuttavia individuare un cut-off di glicemia ben definito, oltre il quale il rischio di eventi avversi aumenta significativamente [2].

In questo studio di coorte longitudinale è stata valutata l'associazione tra glicemia a digiuno e glicemia dopo 1 o 2 ore dal carico orale di glucosio e due delle complicanze più frequenti del GDM, i nati grandi per l'età gestazionale (LGA, peso alla nascita $>90^{\circ}$ centile) e i disordini ipertensivi (HDP, ipertensione gestazionale, preeclampsia, eclampsia).

Tra i nati vivi ad Alberta (318.517), in un periodo di osservazione di circa 6 anni (1 ottobre 2008 - 31 dicembre

$凶$ L. Laviola

luigi.laviola@uniba.it

1 Sezione di Medicina Interna, Endocrinologia, Andrologia e Malattie Metaboliche, Dipartimento dell'Emergenza e dei Trapianti di Organi, Università degli Studi di Bari "Aldo Moro", Bari, Italia
2014), sono stati identificati i nati da gravidanze complicate da GDM (12.942) diagnosticato mediante carico orale di glucosio con $50 \mathrm{~g}$ (GCT) e successivo test di conferma o mediante carico orale di glucosio con 75 g (OGTT), secondo i criteri diagnostici vigenti in Canada.

Tutte le donne con diagnosi di GDM sono state trattate con dieta e modifiche dello stile di vita allo scopo di raggiungere i target glicemici di $95 \mathrm{mg} / \mathrm{dl}, 140 \mathrm{mg} / \mathrm{dl}, 120 \mathrm{mg} / \mathrm{dl} \mathrm{ri}-$ spettivamente a digiuno, 1 ora e 2 ore dopo i pasti. Le donne che superavano questi target sono state trattate con insulina o metformina.

La percentuale di LGA e HDP è stata rispettivamente di 8,1 e 5,1\% nelle donne con GCT negativo, 11,0 e 7,0\% nelle donne con OGTT negativo, 22,4 e 11,9\% nelle donne con elevati valori di glicemia a digiuno e 9,1 e $8,0 \%$ nelle donne con elevati valori glicemici dopo 1 o 2 ore dal carico orale.

Nelle donne con elevati valori di glicemia a digiuno rispetto alle donne con elevati valori dopo carico orale di glucosio, è stato evidenziato un più alto rischio di LGA (OR 2,66, 95\% CI 2,39-2,96) e un più alto rischio di HDP (OR 1,51, 95\% CI 1,33-1,72), indipendentemente dal trattamento farmacologico.

Da un punto di vista fisiopatologico, l'iperglicemia a digiuno potrebbe essere espressione di uno squilibrio metabolico generalizzato, con insulino-resistenza più marcata, associata ad aumentati livelli di acidi grassi liberi e maggiore insulto endotelio-vascolare, con conseguente macrosomia fetale e disordini ipertensivi [3].

Tra i limiti dello studio va considerata la carenza di dati relativi al peso materno pregravidico, un forte fattore di rischio per la LGA e HDP. Inoltre, l'associazione è stata studiata sulla base dei valori glicemici alla diagnosi; mancano, invece, i dati relativi al monitoraggio glicemico domiciliare e i dati circa il raggiungimento dei target glicemici nelle donne in terapia con insulina o metformina. 
Tuttavia, considerata l'elevata numerosità della popolazione esaminata, questo studio aggiunge forza alla già crescente evidenza che l'elevata glicemia a digiuno è un fattore predittivo più importante per LGA e HDP rispetto ai valori post-carico orale di glucosio.

Ulteriori studi prospettici, che valutino anche i dati ricavati dal monitoraggio glicemico domiciliare con e senza terapia farmacologica, potranno verificare se l'iperglicemia a digiuno sia un marker indipendente di un disordine metabolico sottostante, che giustifica il maggior rischio di LGA e HDP.

Funding Note Open access funding provided by Università degli Studi di Bari Aldo Moro within the CRUI-CARE Agreement.

Nota della casa editrice Springer Nature rimane neutrale in riguardo alle rivendicazioni giurisdizionali nelle mappe pubblicate e nelle affiliazioni istituzionali.

Open Access This article is licensed under a Creative Commons Attribution 4.0 International License, which permits use, sharing, adap- tation, distribution and reproduction in any medium or format, as long as you give appropriate credit to the original author(s) and the source, provide a link to the Creative Commons licence, and indicate if changes were made. The images or other third party material in this article are included in the article's Creative Commons licence, unless indicated otherwise in a credit line to the material. If material is not included in the article's Creative Commons licence and your intended use is not permitted by statutory regulation or exceeds the permitted use, you will need to obtain permission directly from the copyright holder. To view a copy of this licence, visit http://creativecommons.org/licenses/by/4.0/.

\section{Bibliografia}

1. Alfadhli EM (2015) Gestational diabetes mellitus. Saudi Med J 36(4):399-406

2. Metzger BE, Lowe LP, Dyer AR et al (2008) Hyperglycemia and adverse pregnancy outcomes. HAPO Study Cooperative Research Group Engl J Med 358(19):1991-2002

3. Metzger BE, Ravnikar V, Vileisis RA, Freinkel N (1982) "Accelerated starvation" and the skipped breakfast in late normal pregnancy. Lancet 1:588-592 